\title{
Mitral valve surgery and coronary artery bypass grafting for moderate-to-severe ischemic mitral regurgitation: Meta-analysis of clinical and echocardiographic outcomes
}

\author{
Sohaib A. Virk, BMed/MD, ${ }^{\text {a }}$ David H. Tian, BMed/MD, ${ }^{\text {a,b }}$ Arunan Sriravindrarajah, BCom (Hons), \\ Douglas Dunn, BE (Hons), ${ }^{\mathrm{a}}$ Hugh D. Wolfenden, MBBS, ${ }^{\mathrm{c}}$ Rakesh M. Suri, MD, DPhil, ${ }^{\mathrm{d}}$ \\ Stine Munkholm-Larsen, MD, ${ }^{a}$ and Christopher Cao, MBBS, BSc, $\mathrm{PhD}^{\mathrm{a}, \mathrm{e}}$
}

\begin{abstract}
Objective: This meta-analysis was conducted to compare clinical and echocardiographic outcomes following isolated coronary artery bypass grafting (CABG) versus $\mathrm{CABG}$ and mitral valve (MV) surgery in patients with moderate-to-severe ischemic mitral regurgitation (IMR).

Methods: Seven databases were systematically searched to identify relevant studies. For eligibility, studies were required to report on the primary endpoint of perioperative or late mortality. Data were analyzed according to predefined clinical endpoints.
\end{abstract}

Results: Four randomized controlled trials $(\mathrm{RCTs})(\mathrm{n}=505)$ and 15 observational studies $(\mathrm{OS})(\mathrm{n}=3785)$ met the criteria for inclusion. Compared with isolated $\mathrm{CABG}$, concomitant CABG and MV surgery was not associated with increased perioperative mortality (RCTs: relative risk [RR] $0.89,95 \%$ confidence interval [CI], 0.26-3.02; OS: RR 1.40, 95\% CI, 0.88-2.23). CABG and MV surgery was associated with significantly lower incidence of moderate-to-severe MR at follow-up (RCTs: RR $0.16,95 \%$ CI, 0.04-0.75; OS: RR $0.20,95 \%$ CI, 0.09-0.48). Late mortality was similar between the surgical approaches in RCTs (hazard ratio [HR] 1.20, $95 \% \mathrm{CI}, 0.57-2.53$ ) and OS (HR 0.99, 95\% CI, 0.81-1.21). There were no significant differences in echocardiographic outcomes. These results remained consistent in subgroup analyses restricted to patients with strictly moderate IMR.

Conclusions: In patients with moderate-to-severe IMR, the addition of MV surgery to $\mathrm{CABG}$ was not associated with increased perioperative mortality. Although concomitant MV surgery reduced recurrence of moderate-to-severe MR at follow-up, this was not associated with a reduction in late mortality. Larger trials with longer follow-up duration are required to further assess longterm survival and freedom from reintervention. (J Thorac Cardiovasc Surg 2017;154:127-36)

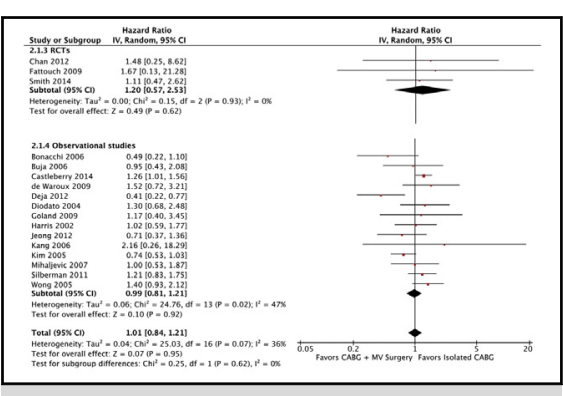

Forest plot for late mortality following CABG versus $\mathrm{CABG}$ and mitral valve surgery.

\section{Central Message}

In patients with moderate-to-severe ischemic mitral regurgitation, there was no difference in perioperative or late mortality following isolated $\mathrm{CABG}$ versus $\mathrm{CABG}$ and mitral valve surgery.

\section{Perspective}

In patients with moderate-to-severe ischemic mitral regurgitation, the addition of mitral valve (MV) surgery to coronary artery bypass grafting was not associated with increased perioperative mortality. Concomitant MV surgery reduced recurrence of moderate-to-severe mitral regurgitation at follow-up, but this was not associated with a reduction in late mortality.

See Editorial Commentary page 137.
Ischemic mitral regurgitation (IMR) is common after myocardial infarction, occurring in up to $50 \%$ of patients. ${ }^{1}$ The development of IMR is associated with an increased

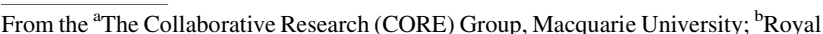
North Shore Hospital; ' Department of Cardiothoracic Surgery, Prince of Wales Hospital; ${ }^{\mathrm{d}}$ Department of Thoracic and Cardiovascular Surgery, Heart and Vascular Institute, Cleveland Clinic, Cleveland, Ohio; and ${ }^{\mathrm{e}}$ Department of Cardiothoracic Surgery, St George Hospital, Sydney, Australia.

Received for publication May 15, 2016; revisions received Feb 5, 2017; accepted for publication March 9, 2017; available ahead of print May 23, 2017.

Address for reprints: Christopher Cao, MBBS, BSc, PhD, The Systematic Review

Unit, The Collaborative Research (CORE) Group, Macquarie University, Sydney,

NSW 2011, Australia (E-mail: drchriscao@gmail.com).

$0022-5223 / \$ 36.00$

Copyright $(2017$ by The American Association for Thoracic Surgery

http://dx.doi.org/10.1016/j.jtcvs.2017.03.039
}

risk of mortality and heart failure, and this risk increases with the severity of regurgitation., ${ }^{2,3}$ In IMR, valvular insufficiency is predominantly due to the adverse left ventricular remodeling and annular dilatation that occurs following myocardial injury, resulting in tethered mitral leaflets with poor coaptation.

Scanning this QR code will take you to the article title page. 


$$
\begin{aligned}
& \text { Abbreviations and Acronyms } \\
& \begin{array}{ll}
\text { CABG } & =\text { coronary artery bypass grafting } \\
\text { CI } & =\text { confidence interval } \\
\text { CPB } & =\text { cardiopulmonary bypass } \\
\text { HR } & =\text { hazard ratio } \\
\text { IMR } & =\text { ischemic mitral regurgitation } \\
\text { LVEF } & =\text { left ventricular ejection fraction } \\
\text { LVESVI } & =\text { left ventricular end-systolic volume } \\
& \text { index } \\
\text { MD } & =\text { mean difference } \\
\text { MV } & =\text { mitral valve } \\
\text { NYHA } & =\text { New York Heart Association } \\
\text { RCT } & =\text { randomized controlled trial } \\
\text { RR } & =\text { relative risk }
\end{array}
\end{aligned}
$$

Isolated coronary artery bypass grafting (CABG) is well-established as the standard treatment for trivial-tomild IMR. ${ }^{4}$ However, the optimal management of moderate-to-severe IMR remains controversial. ${ }^{4,5}$ Revascularization alone may sufficiently restore left ventricular function and dimensions, thereby addressing regurgitation without the potentially increased perioperative risk associated with concomitant mitral valve (MV) surgery. ${ }^{6,7}$ However, it remains unclear whether isolated CABG can achieve similar midterm survival outcomes in patients with moderate-to-severe IMR. ${ }^{8}$ The aim of the present meta-analysis was to compare clinical and echocardiographic outcomes following isolated $\mathrm{CABG}$ and $\mathrm{CABG}$ and MV surgery in patients with at least moderate IMR.

\section{METHODS}

\section{Search Strategy and Study Selection}

Electronic searches were performed using Ovid Medline, Embase, Cochrane Database of Systematic Reviews, Cochrane Central Register of Controlled Trials, Cochrane Methodology Register, Database of Abstracts of Reviews of Effects, and ACP Journal Club from their dates of inception to February 2016. The search terms "mitral" AND ("ischemic" OR "ischaemic") AND ("repair" OR "annuloplasty" OR "replacement") AND ("mortality" OR "death" OR "survival") were entered as keywords and MeSH terms. This was supplemented by hand searching the reference lists of key reviews and all potentially relevant studies.

Two reviewers (A.S. and D.D.) independently screened the title and abstract of records identified in the search. Full-text publications were subsequently reviewed separately if either reviewer considered the manuscript as being potentially eligible. Disagreements regarding final study inclusion were resolved by discussion and consensus.

\section{Eligibility Criteria}

Eligible studies were those reporting perioperative mortality or late survival following isolated $\mathrm{CABG}$ and $\mathrm{CABG}$ and MV surgery in patients with at least moderate IMR. Studies were excluded if they included patients with trivial-to-mild IMR without subgroup analysis by IMR severity.
Studies with fewer than 10 patients in either the isolated CABG or CABG and MV surgery arm were also excluded.

All publications were limited to those involving human subjects and written in English. Abstracts, conference presentations, editorials, and expert opinions were excluded. Review articles were omitted because of potential publication bias and duplication of results. When institutions published duplicate studies with accumulating numbers of patients or increased lengths of follow-up, only the most complete reports were included for quantitative assessment.

\section{Data Extraction}

All data were independently extracted from text, tables, and figures by 2 investigators (A.S. and D.D.). The final results were reviewed by the senior reviewer (C.C.). The predetermined primary endpoints were perioperative mortality and late survival. Perioperative mortality was defined as death within 30 days after surgery or during the same hospitalization. Secondary outcomes included perioperative morbidity, recurrence of moderate-to-severe MR (grade $\geq 2$ ) at latest follow-up, aortic cross-clamp and cardiopulmonary bypass (CPB) durations, New York Heart Association (NYHA) functional status, and echocardiographic measurements.

\section{Statistical Analysis}

Baseline characteristics and operative details were presented as raw values $(\%)$, mean \pm standard deviation or median (interquartile range [IQR]) unless otherwise indicated. The relative risk (RR), mean difference (MD), or hazard ratio (HR) were used as the summary statistics, and reported with $95 \%$ confidence intervals (CIs). When available, adjusted ratios were used from individual studies. Otherwise, unadjusted ratios were computed from the exposure distribution given in the articles. For survival analysis, HRs and associated variance were obtained or calculated from each study by using techniques described by Parmar et $\mathrm{al}^{9}$ and Tierney et al. ${ }^{10}$ When direct calculations were not possible due to a lack of presented data, HRs were estimated using Kaplan-Meier graphs. For analysis of continuous data, data presented as median and IQR were converted to mean and standard deviation using the method of Wan et al. ${ }^{11}$

Meta-analyses were performed by using random-effects models to take into account the anticipated clinical and methodological diversity between studies. The $\mathrm{I}^{2}$ statistic was used to estimate the percentage of total variation across studies due to heterogeneity rather than chance, with values exceeding 50\% indicative of considerable heterogeneity. Mixed-effects meta-regression models were formed by using follow-up duration as a continuous moderator to assess its impact on the incidence of survival and residual MR.

Subgroup analysis was performed by study design to take into account the different levels of evidence provided by observational studies and randomized trials. Sensitivity analysis was performed by IMR severity to specifically assess the impact of concomitant MV surgery in patients with moderate IMR. Publication bias was assessed by using funnel plots comparing log odds ratios with their standard error. The Egger linear regression method and Begg rank correlation test were used to detect funnel plot asymmetry. ${ }^{12,13}$ The Trim-and-Fill method was used to explore the impact of studies potentially missing due to publication bias. ${ }^{14}$ Statistical analysis was conducted with Review Manager Version 5.1.2 (Cochrane Collaboration, Software Update, Oxford, United Kingdom) and publication bias assessed by using Comprehensive Meta-Analysis v2.2 (Biostat Inc, Englewood, NJ). All $P$ values were 2 sided, and values $<.05$ were considered statistically significant.

\section{RESULTS}

A total of 1125 unique records were identified through the database and bibliographic searches. Of these, 1091 


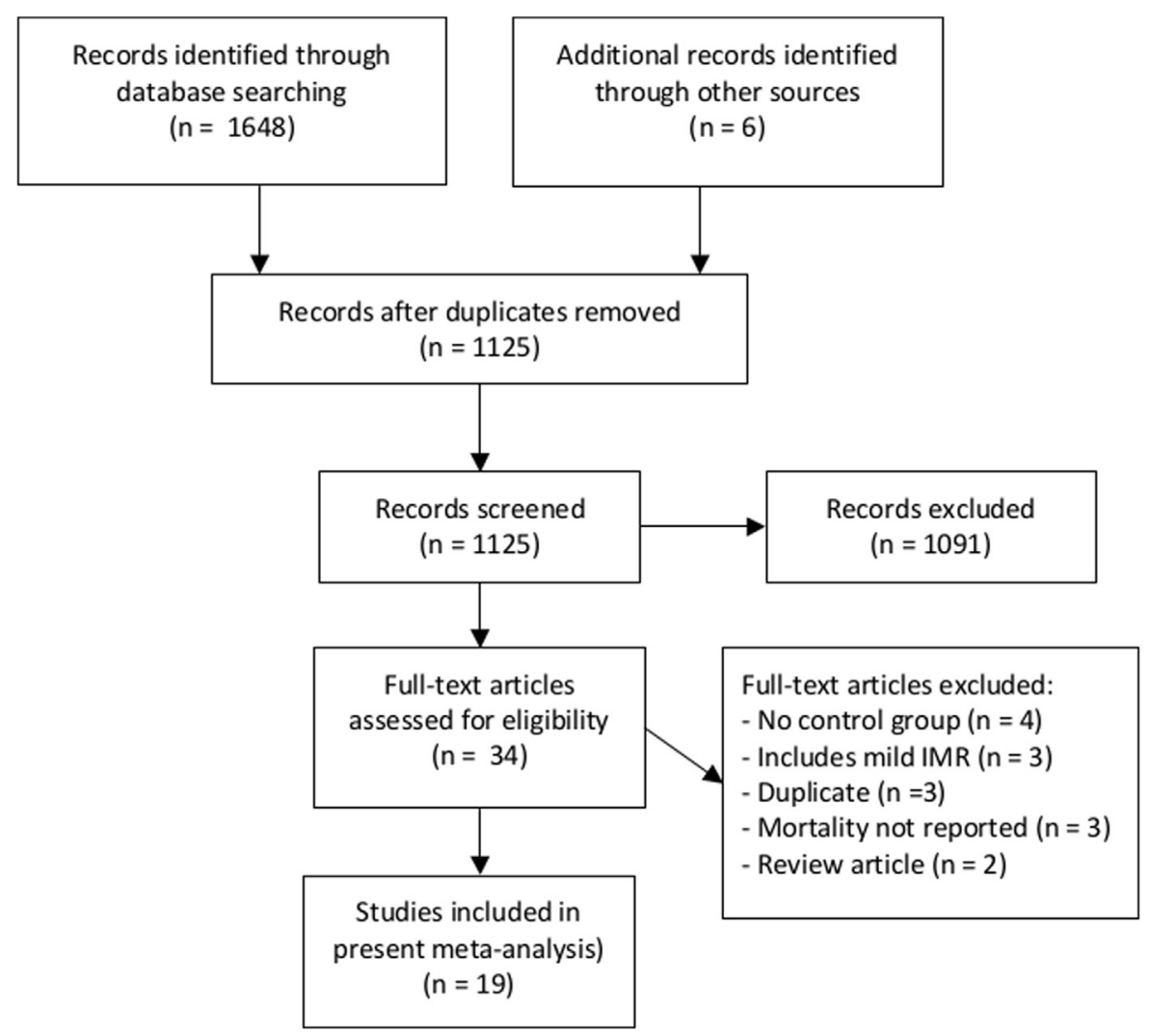

FIGURE 1. PRISMA flow chart for literature search and study selection.

were excluded on the basis of title and abstract content. After screening the full text of the remaining 34 articles, 20 studies met the criteria for inclusion. ${ }^{15-34}$ The study selection process is summarized in Figure 1.

Of the included studies, 4 were randomized controlled trials (RCTs) and the remainder were observational studies. These included data on a total of 4290 patients, including 3038 who underwent isolated CABG and 1252 patients who underwent combined CABG and MV surgery. For patients who underwent MV surgery, all patients included in the RCTs, and most patients included in the observational studies, underwent mitral valve repair. The mean or median follow-up duration ranged from 12 to 87 months. A full summary of study characteristics is presented in Table 1. Baseline demographic and clinical characteristics of study participants are summarized in Table 2.

\section{Intraoperative Outcomes}

In 4 RCTs and 11 observational studies including a total of 1963 patients, CABG and MV surgery was associated with significantly longer CPB (MD 48.12; 95\% CI, 38.05-58.15; $P<.001 ;$ Figure 2, A) and aortic cross-clamp (MD 39.13; 95\% CI, 32.39-45.86; $P<.001$;
Figure 2, $B$ ) durations. These results were consistent in subgroup analyses by study design.

\section{Perioperative Mortality}

In 3 RCTs including a total of 474 patients, concomitant MV surgery was not associated with significantly increased perioperative mortality compared with CABG alone (RR $0.89 ; 95 \%$ CI, 0.26-3.02; $P=.85 ; \mathrm{I}^{2}=0 \%$; Figure $3, A$ ). Likewise, in 14 observational studies including a total of 1975 patients, perioperative mortality was not significantly increased with the addition of concomitant MV surgery (RR $1.40 ; 95 \%$ CI, 0.88-2.23; $P=.16 ; \mathrm{I}^{2}=35 \%$; Figure $3, B$ ).

\section{Perioperative Morbidity}

In 3 RCTs including a total of 203 patients, CABG and MV surgery was not associated with significantly increased risk of stroke (RR 1.65; 95\% CI, 0.27-10.22; $P=.53$; $\left.\mathrm{I}^{2}=0 \%\right){ }^{16-18}$ Likewise, in 2 observational studies including a total of 182 patients, the risk of stroke was not significantly increased with concomitant MV surgery (RR $0.90 ; 95 \%$ CI, $\left.0.23-3.53 ; P=.70 ; \mathrm{I}^{2}=0 \%\right){ }^{20,22}$ In 2 RCTs including a total of 173 patients, the incidence of acute renal failure was not significantly increased with the addition of MV surgery (RR 1.38; 95\% CI, 0.44-4.31; 
TABLE 1. Summary of studies comparing isolated coronary artery bypass graft surgery versus concomitant mitral repair for ischemic mitral regurgitation

\begin{tabular}{|c|c|c|c|c|c|c|c|c|}
\hline \multirow[b]{2}{*}{ Study } & \multirow[b]{2}{*}{$\begin{array}{l}\text { Study } \\
\text { design }\end{array}$} & \multicolumn{2}{|c|}{ No. of patients } & \multirow[b]{2}{*}{$\begin{array}{l}\text { Type of mitral } \\
\text { surgery }\end{array}$} & \multirow[b]{2}{*}{ Follow-up, mo } & \multicolumn{2}{|c|}{ Mean MR grade } & \multirow[b]{2}{*}{$\begin{array}{c}\text { Preoperative MR grade } \\
\text { inclusion criteria }\end{array}$} \\
\hline & & CABG & $\begin{array}{c}\text { CABG + } \\
\text { MVS }\end{array}$ & & & CABG & $\begin{array}{c}\text { CABG }+ \\
\text { MVS }\end{array}$ & \\
\hline Bouchard 2014 & $\mathrm{RCT}$ & 16 & 15 & $\operatorname{MVr}(100 \%)$ & $12^{\mathrm{M}}$ & \multicolumn{2}{|c|}{2 (all patients) } & $2+($ Moderate Only $)$ \\
\hline Smith 2014 & $\mathrm{RCT}$ & 151 & 150 & $\operatorname{MVr}(100 \%)$ & $12^{\mathrm{M}}$ & \multicolumn{2}{|c|}{2 (all patients) } & $2+($ Moderate Only $)$ \\
\hline Chan 2012 & $\mathrm{RCT}$ & 38 & 33 & $\operatorname{MVr}(100 \%)$ & $12^{\mathrm{M}}$ & \multicolumn{2}{|c|}{2 (all patients) } & $2+($ Moderate Only $)$ \\
\hline Fattouch 2009 & $\mathrm{RCT}$ & 54 & 48 & $\operatorname{MVr}(100 \%)$ & $32 \pm 18$ & \multicolumn{2}{|c|}{2 (all patients) } & $2+($ Moderate Only) \\
\hline Castleberry 2014 & OS & 1651 & 243 & $\begin{array}{l}\text { MVR or MVr } \\
\quad(\% \mathrm{NR})\end{array}$ & $64.4^{\mathrm{M}}$ & $2.25^{*}$ & $3.46^{*}$ & $\geq 2+($ Moderate to Severe $)$ \\
\hline Sa 2013 & OS & 26 & 16 & MVR (100\%) & NR & NR & NR & NS (Moderate to Severe) \\
\hline Deja 2012 & OS & 42 & 49 & $\begin{array}{l}\operatorname{MVr}(98 \%) \\
\operatorname{MVR}(2 \%)\end{array}$ & $56^{\mathrm{M}}$ & NR & NR & NS (Moderate to Severe) \\
\hline Jeong 2012 & OS & 77 & 63 & $\operatorname{MVr}(100 \%)$ & $44 \pm 34.5$ & NR & NR & $3+($ Moderate Only $)$ \\
\hline Silberman 2011 & OS & 108 & 87 & $\operatorname{MVr}(100 \%)$ & $87 \pm 50$ & $3.1 \pm 0.3^{*}$ & $3.8 \pm 0.4^{*}$ & $\geq 3+($ Moderate to Severe $)$ \\
\hline de Waroux 2009 & OS & 42 & 34 & $\operatorname{MVr}(100 \%)$ & $51 \pm 44$ & $2.2 \pm 0.4$ & $2.4 \pm 0.5$ & $\geq 3+($ Moderate to Severe $)$ \\
\hline Goland 2009 & OS & 55 & 28 & $\operatorname{MVr}(100 \%)$ & $61.2 \pm 43.2$ & \multicolumn{2}{|c|}{3 (all patients) } & $3+($ Moderate Only) \\
\hline Mihaljevic 2007 & OS & 54 & 54 & $\operatorname{MVr}(100 \%)$ & $48^{\mathrm{M}}$ & 3.24 & 3.19 & $\geq 3+($ Moderate to Severe $)$ \\
\hline Bonacchi 2006 & OS & 36 & 40 & $\operatorname{MVr}(100 \%)$ & $32.4 \pm 11$ & $2.5 \pm 0.5^{*}$ & $3.3 \pm 0.6^{*}$ & $\geq 2+($ Moderate to Severe $)$ \\
\hline Buja 2006 & OS & 50 & 39 & $\begin{array}{l}\operatorname{MVR}(56 \%) ; \\
\operatorname{MVr}(44 \%)\end{array}$ & $34.9[5.3-104]$ & NR & NR & $\geq 3+($ Moderate to Severe $)$ \\
\hline Kang 2006 & OS & 57 & 50 & $\operatorname{MVr}(100 \%)$ & $\begin{array}{c}41 \pm 27(\mathrm{CABG}) \\
37 \pm 22 \\
(\mathrm{MVr}+\mathrm{CABG})\end{array}$ & $2.5 \pm 0.5$ & $2.8 \pm 0.4$ & $2+/ 3+$ (Moderate to Severe) \\
\hline Kim 2005 & OS & 168 & 187 & $\operatorname{MVr}(100 \%)$ & NR & $2.63^{*}$ & $3.81 *$ & $\geq 3+($ Moderate to Severe $)$ \\
\hline Wong 2005 & OS & 220 & 31 & $\operatorname{MVr}(100 \%)$ & $51.6^{\mathrm{m}}$ & $2.3 \pm 0.7^{*}$ & $3.1 \pm 0.3^{*}$ & $3+$ (Moderate Only) \\
\hline Diodato 2004 & OS & 51 & 51 & $\operatorname{MVr}(100 \%)$ & $\begin{array}{c}39 \pm 25(\mathrm{CABG}) \\
37 \pm 25 \\
(\mathrm{MVr}+\mathrm{CABG})\end{array}$ & NR & NR & $\geq 2+($ Moderate to Severe $)$ \\
\hline Harris 2002 & OS & 142 & 34 & $\begin{array}{l}\operatorname{MVr}(85 \%) ; \\
\quad \operatorname{MVR}(15 \%)\end{array}$ & $\begin{array}{c}61.2 \pm 3.6(\mathrm{CABG}) \\
56.4 \pm 7.2 \\
(\mathrm{MVS}+\mathrm{CABG})\end{array}$ & $2.1 \pm 0.3^{*}$ & $2.6 \pm 0.5^{*}$ & $2+/ 3+($ Moderate Only $)$ \\
\hline
\end{tabular}

Data presented as n (\%), mean \pm standard deviation, or median [interquartile range], unless stated otherwise. $C A B G$, Coronary artery bypass graft; $M V S$, mitral valve surgery; $M R$, mitral regurgitation; $R C T$, randomized controlled trial; $M V r$, mitral valve repair; $O S$, observational study; $M V R$, mitral valve replacement; $N R$, not reported; $N S$, not specified; $M$, median; $m$, mean. $* P<.05$.

$\left.P=.58 ; \mathrm{I}^{2}=0 \%\right) .{ }^{17,18}$ Similarly, in 3 observational studies including a total of 433 patients, there was no significant difference in acute renal failure between the 2 groups (RR $1.08 ; 95 \%$ CI, 0.28-4.19;P $\left.=.91 ; \mathrm{I}^{2}=79 \%\right)^{20,22,31}$ Meta-analyses could not be performed for other morbidity endpoints due to the lack of outcome reporting across studies.

\section{Survival}

In 3 RCTs involving a total of 462 patients, there was no difference in survival at follow-up between patients who underwent isolated $\mathrm{CABG}$ and those who underwent CABG and MV surgery (HR 1.20; 95\% CI, 0.57-2.53; $P=.62 ; \mathrm{I}^{2}=0 \%$; Figure $\left.4, A\right)$. Similarly, in 14 observational studies involving a total of 3828 patients, the 2 surgical cohorts had similar survival at the time of latest follow-up (HR 0.99; 95\% CI, 0.81-1.21; $P=.92$; $\mathrm{I}^{2}=47 \%$; Figure $\left.4, B\right)$. Aggregated survival was calculated by using either Kaplan-Meier curves assuming constant censoring $^{18,28}$ or with censoring information, ${ }^{19,23-25,27,34}$ events and $P$ value, ${ }^{17,22,26,29,30}$ adjusted $\mathrm{HR}{ }^{21}$ or unadjusted HR, ${ }^{16,31}$ as per methods described by Tierney and colleagues. ${ }^{10}$

\section{Recurrence of Moderate-to-Severe Mitral Regurgitation}

In 4 RCTs including a total of 459 patients, CABG and MV surgery was associated with significantly lower incidence of moderate-to-severe (grade $\geq 2$ ) regurgitation at latest follow-up (RR 0.16; 95\% CI, 0.04-0.75; $P=.02$; $\mathrm{I}^{2}=68 \%$; Figure $\left.5, A\right)$. This result was also demonstrated in an analysis of 4 observational studies including a total of 312 patients (RR 0.25; 95\% CI, 0.12-0.50; $P<.001$; $\mathrm{I}^{2}=21 \%$; Figure $\left.5, B\right)$.

\section{Echocardiographic Outcomes}

Due to limited data from observational studies, meta-analyses of echocardiographic outcomes were limited to RCTs. In 3 RCTs including 402 patients, CABG and MV surgery was not associated with significantly greater 
TABLE 2. Baseline characteristics of participants in studies comparing isolated coronary artery bypass graft surgery versus concomitant mitral repair for ischemic mitral regurgitation

\begin{tabular}{|c|c|c|c|c|c|c|c|c|c|c|c|c|c|c|}
\hline \multirow[b]{2}{*}{ Study } & \multicolumn{2}{|c|}{ Age } & \multicolumn{2}{|c|}{ Male (\%) } & \multicolumn{2}{|c|}{ HTN (\%) } & \multicolumn{2}{|c|}{ Diabetes $(\%)$} & \multicolumn{2}{|c|}{$\mathbf{A F}(\%)$} & \multicolumn{2}{|c|}{ Prior MI $(\%)$} & \multicolumn{2}{|c|}{ LVEF \% } \\
\hline & CABG & $\begin{array}{c}\text { CABG }+ \\
\text { MVr }\end{array}$ & CABG & $\begin{array}{c}\text { CABG + } \\
\text { MVr }\end{array}$ & CABG & $\begin{array}{c}\text { CABG }+ \\
\text { MVr }\end{array}$ & CABG & $\begin{array}{c}\text { CABG }+ \\
\text { MVr }\end{array}$ & CABG & $\begin{array}{c}\text { CABG + } \\
\text { MVr }\end{array}$ & CABG & $\begin{array}{c}\text { CABG + } \\
\text { MVr }\end{array}$ & CABG & $\begin{array}{c}\text { CABG }+ \\
\text { MVr }\end{array}$ \\
\hline $\begin{array}{c}\text { Bouchard } \\
2014\end{array}$ & $65 \pm 12$ & $69 \pm 7$ & 88 & 75 & 56 & 73 & 50 & 27 & 13 & 20 & 75 & 60 & $41.5 \pm 17.4$ & $45.7 \pm 11.4$ \\
\hline Smith 2014 & $65.2 \pm 11.3$ & $64.3 \pm 9.6$ & 66 & 71 & NR & NR & 44 & 51 & $23^{*}$ & $13 *$ & 64 & 69 & $41.2 \pm 11.6$ & $39.3 \pm 10.9$ \\
\hline Chan 2012 & $70.4 \pm 7.9$ & $70.9 \pm 10.5$ & 74 & 74 & 59 & 50 & 38 & 35 & 10 & 6 & 72 & 74 & $40.3 \pm 16.1$ & $40.0 \pm 17.3$ \\
\hline $\begin{array}{c}\text { Fattouch } \\
2009\end{array}$ & $66 \pm 7$ & $64 \pm 9$ & 65 & 63 & 43 & 54 & 59 & 58 & NR & NR & 100 & 100 & $43 \pm 9$ & $42 \pm 10$ \\
\hline $\begin{array}{c}\text { Castleberry } \\
2014\end{array}$ & $66(58-73)^{*}$ & $66(58-74)^{*}$ & 65 & 54 & 69 & 61 & 36 & 35 & NR & NR & NR & NR & $48(35-60)^{*}$ & $45(31-60)^{*}$ \\
\hline Sa 2013 & NR & NR & 54 & 56 & 85 & 94 & 42 & 25 & NR & NR & 46 & 50 & $47 \pm 3$ & $45 \pm 5$ \\
\hline Deja 2012 & $64(56-70)$ & $60(54-68)$ & 88 & 90 & 50 & 59 & $55^{*}$ & $27 *$ & NR & NR & 74 & 82 & $30(25-35)^{*}$ & $22(19-28)^{*}$ \\
\hline Jeong 2012 & $65.4 \pm 9.1$ & $63.9 \pm 9.1$ & 69 & 83 & 69 & 59 & 46 & 49 & $4^{*}$ & $18^{*}$ & 100 & 100 & $41.5 \pm 12.3$ & $38.3 \pm 12.0$ \\
\hline $\begin{array}{l}\text { Silberman } \\
2011\end{array}$ & $68 \pm 9^{*}$ & $63 \pm 10^{*}$ & 70 & 78 & 64 & 61 & 44 & 53 & 12 & 7 & NR & NR & NR & NR \\
\hline $\begin{array}{l}\text { de Waroux } \\
2009\end{array}$ & $61.4 \pm 10.1$ & $64.0 \pm 8.4$ & 79 & 76 & 29 & 24 & 24 & 41 & NR & NR & NR & NR & $34.4 \pm 10.1$ & $33.0 \pm 12.5$ \\
\hline $\begin{array}{r}\text { Goland } \\
2009\end{array}$ & $69 \pm 11$ & $68 \pm 9$ & 64 & 80 & 65 & 61 & 36 & 29 & NR & NR & 44 & 61 & $39 \pm 11$ & $37 \pm 11$ \\
\hline $\begin{array}{c}\text { Mihaljevic } \\
2007\end{array}$ & $66 \pm 9.2$ & $66 \pm 9.6$ & 59 & 69 & 70 & 71 & 42 & 32 & 0 & 0 & 89 & 83 & NR & NR \\
\hline $\begin{array}{c}\text { Bonacchi } \\
2006\end{array}$ & $64.5 \pm 6$ & $64.6 \pm 6$ & 70 & 57 & 53 & 63 & 38 & 41 & NR & NR & NR & NR & $27 \pm 5$ & $27.6 \pm 5$ \\
\hline Buja 2006 & $75 \pm 7.4$ & $72 \pm 9.1$ & 64 & 62 & 72 & 74 & 42 & 41 & 38 & 31 & 88 & 85 & $38 \pm 13^{*}$ & $50 \pm 14^{*}$ \\
\hline Kang 2006 & $63 \pm 9$ & $61 \pm 10$ & 68 & 74 & NR & NR & 56 & 52 & 0 & 12 & NR & NR & $36 \pm 9$ & $36 \pm 11$ \\
\hline Kim 2005 & $71 \pm 11$ & $72 \pm 9$ & 67 & 71 & 64 & 62 & 40 & 40 & NR & NR & $48^{*}$ & $63 *$ & $43 \pm 15^{*}$ & $38 \pm 14^{*}$ \\
\hline Wong 2005 & NR & NR & NR & NR & NR & NR & NR & NR & NR & NR & NR & NR & $42.2 \pm 15.3$ & $39.0 \pm 13.6$ \\
\hline $\begin{array}{c}\text { Diodato } \\
2004\end{array}$ & $69 \pm 11^{*}$ & $65 \pm 10^{*}$ & 51 & 47 & 69 & 82 & NR & NR & NR & NR & 67 & 78 & $31 \pm 11$ & $35 \pm 12$ \\
\hline Harris 2002 & $68.8 \pm 9.8$ & $65.6 \pm 10.8$ & 54 & 44 & 76 & 68 & 46 & 50 & NR & NR & 80 & 76 & $38.7 \pm 12.6$ & $38.0 \pm 13.8$ \\
\hline
\end{tabular}

increase in left ventricular ejection fraction (LVEF) at follow-up (MD 0.19; 95\% CI, -3.26 to 3.65; $P=.91){ }^{15,16,18}$ In 2 RCTs including 339 patients, the decrease in left ventricular end-systolic volume index (LVESVI) was not significantly greater following CABG and MV surgery (weighted mean difference [WMD] $-3.59 ; 95 \% \mathrm{CI},-17.98$ to $10.81 ; P=.63) .{ }^{16,17}$ In 2 RCTs including 88 patients, the decrease in residual regurgitant volume was not significantly different between patients who underwent CABG and MV surgery and those who underwent isolated CABG (WMD - 4.78; 95\% CI, -26.61 to $\left.17.06 ; P=.67 ; \mathrm{I}^{2}=94 \%\right) .{ }^{15,17}$

\section{NYHA Functional Status}

In 3 RCTs including a total of 330 patients, the proportion of patients in NYHA class III/IV at follow-up were similar following isolated $\mathrm{CABG}$ or $\mathrm{CABG}$ and $\mathrm{MV}$ surgery (RR $0.67 ; 95 \%$ CI, $\left.0.25-1.82 ; P=.43 ; \mathrm{I}^{2}=18 \%\right) .{ }^{15-17}$ This result was also demonstrated in an analysis of 4 observational studies including a total of 292 patients (RR $1.23 ; 95 \%$ CI, $\left.0.78-1.93 ; P=.37 ; \mathrm{I}^{2}=5 \%\right)^{26,27,33,35}$

\section{Subgroup Analysis: Moderate MR}

Subgroup analysis was conducted to assess studies in which all patients had only moderate IMR. This included 4 RCTs and 4 observational studies, reporting on a total of 1155 patients. ${ }^{15-18,22,25,31,34}$ Within this group, combined CABG and MV surgery was found to be associated with significantly increased perioperative mortality in observational studies (RR 1.81;95\% CI, $1.03-3.15 ; P=.02 ; \mathrm{I}^{2}=0 \%$ ) but not within RCTs (RR $0.89 ; 95 \%$ CI, 0.26-3.02; $P=.85 ; \mathrm{I}^{2}=0 \%$ ). Patients who underwent combined CABG and MV surgery still had significantly reduced recurrence of $\mathrm{MR}$ at latest follow-up in both RCTs (RR 0.16; 95\% CI, 0.04-0.75; $P<.001 ; \mathrm{I}^{2}=68 \%$ ) and observational studies (RR 0.26; $95 \%$ CI, 0.11-0.65; $P=.004 ; \mathrm{I}^{2}=38 \%$ ). There remained no difference between the surgical approaches with regard 




A

\begin{tabular}{|c|c|c|c|c|c|c|c|c|}
\hline \multirow[b]{2}{*}{ Study or Subgroup } & \multicolumn{3}{|c|}{ CABG + MV Surgery } & \multicolumn{3}{|c|}{ Isolated CABG } & \multirow[b]{2}{*}{ Weight } & \multirow{2}{*}{$\begin{array}{l}\text { Mean Difference } \\
\text { IV, Random, } 95 \% \mathrm{CI}\end{array}$} \\
\hline & Mean & SD & Total & Mean & SD & Total & & \\
\hline \multicolumn{9}{|l|}{ 1.7.1 RCTs } \\
\hline Bouchard 20 & 94 & 28 & 15 & 63 & 29 & 16 & $5.0 \%$ & $31.00[10.93,51.0$ \\
\hline Chan & 73.75 & 14.98 & 33 & 36.75 & 10.47 & 38 & $8.3 \%$ & $37.00[30.90,43.10$ \\
\hline Fattouch 2009 & 88 & 19 & 48 & 38 & 8 & 54 & $8.3 \%$ & $50.00[44.22,55.78$ \\
\hline $\begin{array}{l}\text { Smith } 2014 \\
\text { Subtotal (95\% Cl) }\end{array}$ & 117.2 & 35.4 & $\begin{array}{l}150 \\
246\end{array}$ & 74.7 & 36.7 & $\begin{array}{l}151 \\
259\end{array}$ & $\begin{array}{r}7.9 \% \\
29.4 \%\end{array}$ & $\begin{array}{l}42 . \\
41.9\end{array}$ \\
\hline \multicolumn{9}{|c|}{$\begin{array}{l}\text { Heterogeneity: } \text { Tau }^{2}=40.68 ; \mathrm{Chi}^{2}=10.73, \mathrm{df}=3(\mathrm{P}=0.01) ; \mathrm{I}^{2}=72 \% \\
\text { Test for overall effect: } Z=10.63(\mathrm{P}<0.00001)\end{array}$} \\
\hline \multicolumn{9}{|c|}{ 1.7.2 Observational Studies } \\
\hline Bonacchi 2006 & 90 & 18 & 40 &  & . & 36 & $7.9 \%$ & \\
\hline Buja & 111 & 22 & 39 & 68 & 115 & 50 & $2.9 \%$ & 43. \\
\hline de Waroux 2 & 118 & 31.6 & 34 & 73.3 & 26.7 & 42 & $6.6 \%$ & $44.70[31.36,58.0$ \\
\hline Deja 2012 & 93.67 & 36.6 & 49 & 59.67 & 22.4 & 42 & $6.8 \%$ & $34.00[21.72,46.2$ \\
\hline Diodato 2004 & 112 & 33 & 51 & 87 & 39 & 51 & $6.4 \%$ & $25.00[10.98,39.02$ \\
\hline Goland 2009 & 114 & 30 & 28 & 60 & 36 & 55 & $6.3 \%$ & $54.00[39.37,68.6]$ \\
\hline Harris 2002 & 123 & 49 & 34 & 63 & 27 & 142 & $5.7 \%$ & $60.00[42.94,77.06$ \\
\hline Kang 2006 & 107 & 53 & 50 & 67 & 59 & 57 & $4.7 \%$ & $40.00[18.78,61.22$ \\
\hline Kim 2005 & 114 & 30 & 187 & 60 & 48 & 168 & $7.8 \%$ & $54.00[45.56,62.4$ \\
\hline Mihaljevic 200 & 97 & 24 & 54 & 74 & 28 & 54 & $7.5 \%$ & $23.00[13.16,32.84$ \\
\hline $\begin{array}{l}\text { Silberman } 2011 \\
\text { Subtotal }(95 \% \mathrm{Cl})\end{array}$ & 92 & 24 & $\begin{array}{r}87 \\
653\end{array}$ & 53 & 25 & $\begin{array}{l}108 \\
805\end{array}$ & $\begin{array}{r}8.1 \% \\
70.6 \%\end{array}$ & $\begin{array}{l}39.00[32.10,45.90 \\
38.53[29.08,47.98\end{array}$ \\
\hline
\end{tabular}

Heterogeneity: $\mathrm{Tau}^{2}=202.15 ; \mathrm{Ch}^{2}=70.10$, df $=10(\mathrm{P}<0.00001) ; \mathrm{I}^{2}=86 \%$

Test for overall effect: $Z=7.99(P<0.00001)$

Total $(95 \% \mathrm{Cl})$

899

$1064 \quad 100.0 \% 39.13[32.39,45.86]$

Heterogeneity: $\mathrm{Tau}^{2}=133.00 ; \mathrm{Chi}^{2}=88.71, \mathrm{df}=14(\mathrm{P}<0.00001) ; \mathrm{I}^{2}=84 \%$

Test for overall effect: $Z=11.39(P<0.00001)$

Test for subgroup differences: $C \mathrm{Ch}^{2}=0.30, \mathrm{df}=1(\mathrm{P}=0.59), \mathrm{I}^{2}=0 \%$

Favors $C A B C+$ MV Surgery Favors Isolated CABC

B

FIGURE 2. Forest plot displaying the mean difference (MD) in aortic cross-clamp (2A) and cardiopulmonary bypass ( $C P B)$ durations (2B) for isolated coronary artery bypass graft $(C A B G)$ surgery versus $\mathrm{CABG}$ and mitral valve $(M V)$ surgery. The MD in each study corresponds to the middle of the squares, the horizontal lines show the $95 \%$ confidence intervals $(C I S)$, and the pooled MD is represented by the middle of the solid diamond. A test of heterogeneity between studies is given below the summary statistics. $S D$, Standard deviation; $R C T$, randomized controlled trial.

to long-term survival (HR 1.09; 95\% CI, 0.84-1.42; $P=.53 ; \mathrm{I}^{2}=0 \%$ ) or NYHA functional status (RR 1.16; $95 \%$ CI, $0.61-2.21 ; P=.65 ; \mathrm{I}^{2}=37 \%$ ).

\section{Meta-Regression Analyses}

Meta-regression analyses demonstrated that duration of follow-up was not a significant moderator for long-term survival $(P=.60)$ and residual MR $(P=.20)$. Meta-regression analyses could not be performed for other endpoints due to the paucity of studies.

\section{Publication Bias}

Neither the Begg rank correlation test $(P=.51)$ nor Egger linear regression method $(P=.40)$ suggested publication bias was a significant factor when perioperative mortality was selected as an endpoint. Likewise, 


\begin{tabular}{|c|c|c|c|c|c|c|c|}
\hline \multirow{2}{*}{$\begin{array}{l}\text { Study or Subgroup } \\
1.1 .1 \mathrm{RCTs}\end{array}$} & \multicolumn{2}{|c|}{ CABG + MV Surgery } & \multicolumn{2}{|c|}{ Isolated CABG } & \multirow[t]{2}{*}{ Weight } & $\begin{array}{c}\text { Risk Ratio } \\
\mathrm{M}-\mathrm{H}, \text { Random, } 95 \% \mathrm{Cl}\end{array}$ & $\begin{array}{c}\text { Risk Ratio } \\
\text { M-H, Random, } 95 \% \mathrm{Cl} \\
\end{array}$ \\
\hline & & & & & & & \\
\hline Chan 2012 & 1 & 33 & 1 & 38 & $20.1 \%$ & $1.15[0.07,17.70]$ & $=$ \\
\hline Fattouch 2009 & 2 & 48 & 1 & 54 & $26.8 \%$ & $2.25[0.21,24.04]$ & \\
\hline $\begin{array}{l}\text { Smith } 2014 \\
\text { Subtotal }(95 \% \mathrm{CI})\end{array}$ & 2 & $\begin{array}{l}150 \\
231\end{array}$ & 4 & $\begin{array}{l}151 \\
243\end{array}$ & $\begin{array}{r}53.1 \% \\
100.0 \%\end{array}$ & $\begin{array}{l}0.50[0.09,2.71] \\
0.89[0.26,3.02]\end{array}$ & \\
\hline $\begin{array}{l}\text { Total events } \\
\text { Heterogeneity: Tau }{ }^{2} \\
\text { Test for overall effec }\end{array}$ & $\begin{array}{l}{ }^{5} \\
0.00 ; \mathrm{Chi}^{2} \\
\mathrm{Z}=0.19(\mathrm{P}\end{array}$ & 5) & $(P=0$. & 9); $1^{2}=$ & & & \\
\hline
\end{tabular}

A

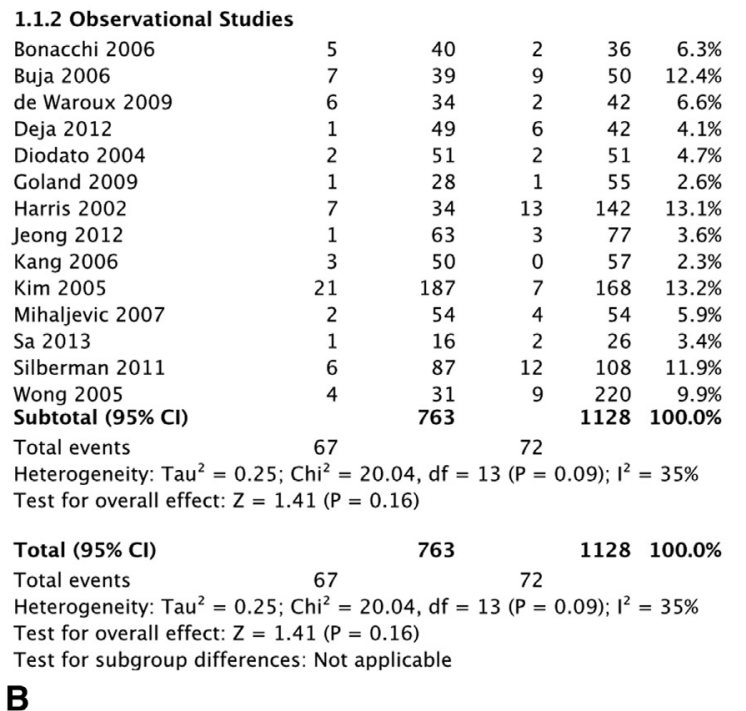

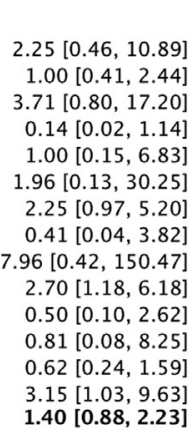

$1.40[0.88,2.23]$

$1.40[0.88,2.23]$

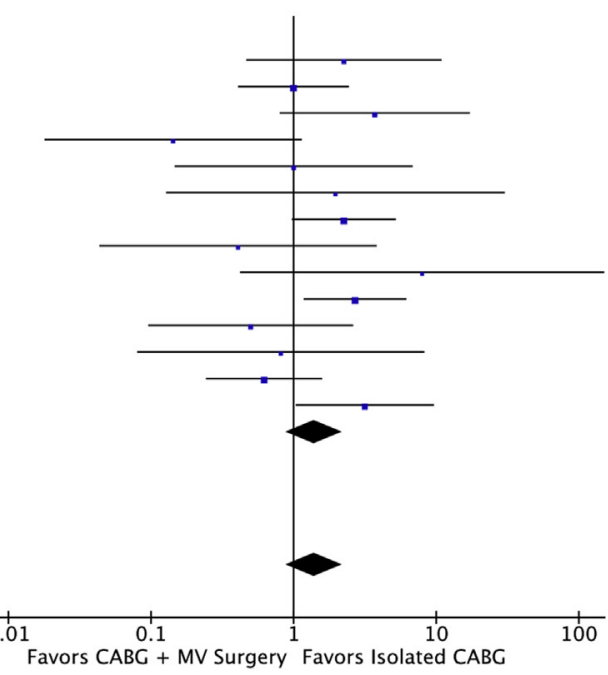

FIGURE 3. Forest plot displaying relative risk (RR) for perioperative mortality following isolated coronary artery bypass graft (CABG) surgery versus CABG and mitral valve $(M V)$ surgery in randomized controlled trials (3A) and observational studies (3B). The RR of individual studies correspond to the middle of the squares, the horizontal lines show the $95 \%$ confidence intervals $(C I s)$, and the pooled RR is represented by the middle of the solid diamond. A test of heterogeneity between studies is given below the summary statistics. $R C T$, Randomized controlled trial.

publication bias was not found to significantly influence results for late mortality (Begg rank correlation test, $P=.97$; Egger linear regression method, $P=.51$ ).

\section{DISCUSSION}

Despite the prevalence of IMR in patients referred for surgical revascularization and its association with poorer clinical outcomes, the optimal management of moderateto-severe IMR remains unclear. ${ }^{3}$ The present metaanalysis assessed the safety and efficacy of isolated CABG versus combined CABG and MV surgery in patients with moderate-to-severe IMR. Concomitant MV surgery was not associated with an increase in perioperative mortality. However, the addition of MV surgery to CABG resulted in significantly reduced recurrence of moderate-to-severe MR at latest follow-up. There was no significant difference in late mortality or NYHA functional status between patients who underwent isolated CABG or those who underwent $\mathrm{CABG}$ and MV surgery. These findings were replicated in subgroup analysis conducted to assess the impact of concomitant MV surgery in patients with strictly moderate IMR.
CPB and aortic cross-clamp durations were found to be significantly higher in patients undergoing $\mathrm{CABG}+\mathrm{MV}$ surgery. This is an intuitive finding given the increased complexity of a combined procedure. Longer $\mathrm{CPB}$ and aortic cross-clamp durations have been linked with a number of complications, including microemboli, increased transfusion requirements, coagulation defects, and immunosuppression. ${ }^{35-37}$ In the present meta-analysis, increased operative durations did not translate to an increase in adverse perioperative events. However, it must be emphasized that the present study was unable to assess key perioperative morbidity outcomes, such as major bleeding, reoperation, myocardial infarction, and wound infection. In particular, there were a very limited number of studies assessing neurological events and the event rate may have been too low to enable sufficiently powered analysis. Indeed, in 2 of the included RCTs, the point estimate for stroke suggested a possible trend toward increased risk following combined $\mathrm{CABG}+\mathrm{MV}$ surgery. ${ }^{16,17}$ As such, given the paucity of endpoints reported in current studies, it is not possible to exclude an increased risk of perioperative morbidity with the addition of concomitant MV surgery. 


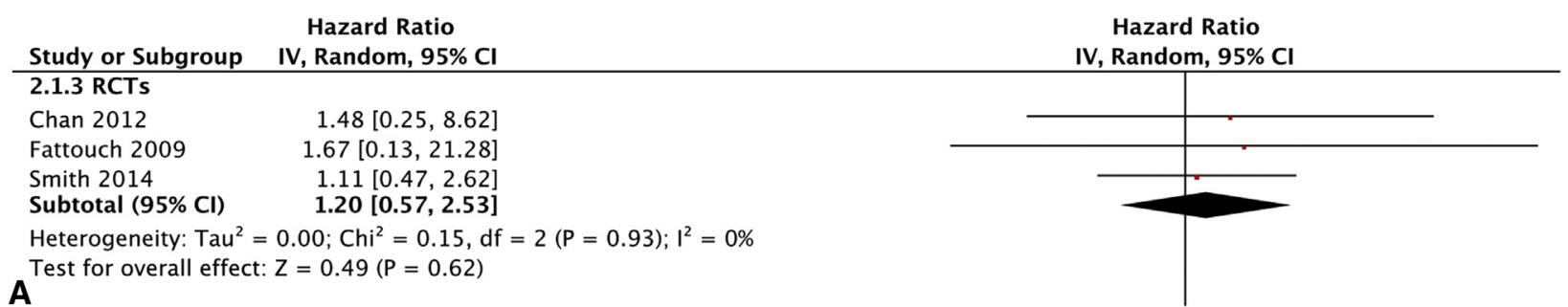

\begin{tabular}{|c|c|}
\hline \multicolumn{2}{|c|}{ 2.1.4 Observational studies } \\
\hline Bonacchi 2006 & $0.49[0.22,1.10]$ \\
\hline Buja 2006 & $0.95[0.43,2.08]$ \\
\hline Castleberry 2014 & $1.26[1.01,1.56]$ \\
\hline de Waroux 2009 & $1.52[0.72,3.21]$ \\
\hline Deja 2012 & $0.41[0.22,0.77]$ \\
\hline Diodato 2004 & $1.30[0.68,2.48]$ \\
\hline Goland 2009 & $1.17[0.40,3.45]$ \\
\hline Harris 2002 & $1.02[0.59,1.77]$ \\
\hline Jeong 2012 & $0.71[0.37,1.36]$ \\
\hline Kang 2006 & $2.16[0.26,18.29]$ \\
\hline Kim 2005 & $0.74[0.53,1.03]$ \\
\hline Mihaljevic 2007 & $1.00[0.53,1.87]$ \\
\hline Silberman 2011 & $1.21[0.83,1.75]$ \\
\hline $\begin{array}{l}\text { Wong } 2005 \\
\text { Subtotal }(95 \% \mathrm{CI})\end{array}$ & $\begin{array}{l}1.40[0.93,2.12] \\
0.99[0.81,1.21]\end{array}$ \\
\hline \multicolumn{2}{|c|}{$\begin{array}{l}\text { Heterogeneity: } \text { Tau }^{2}=0.06 ; \mathrm{Chi}^{2}=24.76, \mathrm{df}=13(\mathrm{P}=0.02) ; \mathrm{I}^{2}=47 \% \\
\text { Test for overall effect: } Z=0.10(P=0.92)\end{array}$} \\
\hline Total $(95 \% \mathrm{CI})$ & $1.01[0.84,1.21]$ \\
\hline \multicolumn{2}{|c|}{$\begin{array}{l}\text { Heterogeneity: } \text { Tau }^{2}=0.04 ; \mathrm{Chi}^{2}=25.03, \mathrm{df}=16(\mathrm{P}=0.07) ; \mathrm{I}^{2}=36 \% \\
\text { Test for overall effect: } \mathrm{Z}=0.07(\mathrm{P}=0.95) \\
\text { Test for subgroup differences: } \mathrm{Chi}^{2}=0.25, \mathrm{df}=1(\mathrm{P}=0.62), \mathrm{I}^{2}=0 \%\end{array}$} \\
\hline
\end{tabular}

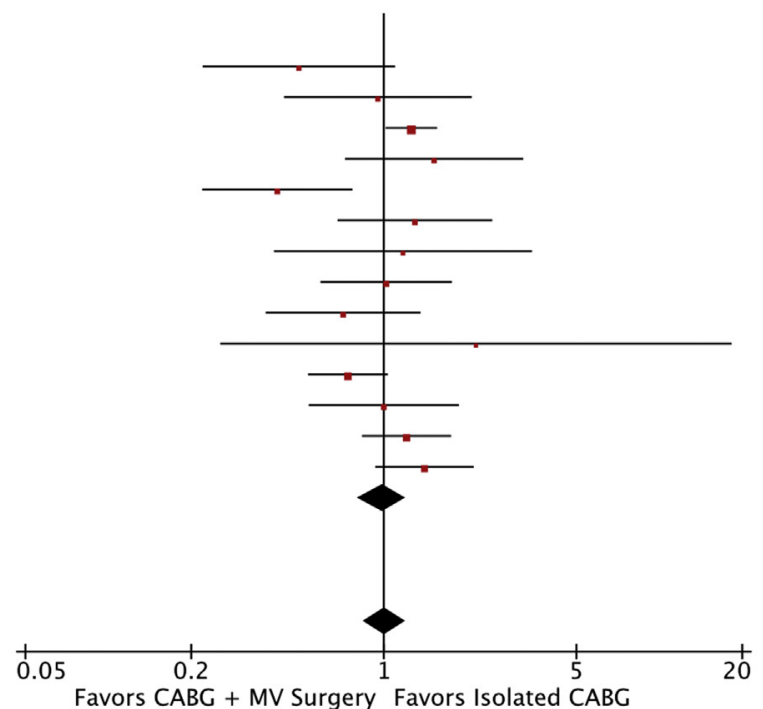

FIGURE 4. Forest plot displaying hazard ratio (HR) for late mortality following isolated coronary artery bypass graft ( $C A B G)$ surgery versus CABG and mitral valve $(M V)$ surgery in randomized controlled trials (4A) and observational studies (4B). The HR of individual studies correspond to the middle of the squares, the horizontal lines show the $95 \%$ confidence intervals $(C I s)$, and the pooled HR is represented by the middle of the solid diamond. A test of heterogeneity between studies is given below the summary statistics. $R C T$, Randomized controlled trial; $S E$, standard error.

Key findings from the present study demonstrated that the proportion of patients with residual MR of at least moderate severity was significantly lower following concomitant mitral intervention. However, this was not associated with any significant advantage with regard to survival or functional status at the time of latest follow-up. This represents an unexpected finding given the association previously demonstrated between persistent MR and increased mortality ${ }^{38}$ It is possible the limited sample size and follow-up durations of the included studies, particularly the RCTs, may not have provided sufficient time or statistical power to highlight the clinical benefit of reduced residual MR. Alternatively, surgical revascularization alone may have sufficiently improved adverse left ventricular remodeling and contractility so as to mitigate the adverse impact of residual MR. ${ }^{6,7}$ The latter suggestion was supported by our meta-analysis of echocardiographic outcomes, which demonstrated similar pooled LVEF and LVESVI in patients who underwent either isolated CABG or CABG and MV surgery. Nonetheless, larger randomized trials with longer follow-up durations are required to clarify this discrepancy between echocardiographic and clinical outcomes.

The present meta-analysis highlighted the considerable variability that exists with regard to the definition of "moderate" IMR. Although the American Society of Echocardiography and American Heart Association/ American College of Cardiology have proposed standardized guidelines for the determination of MR grade, ${ }^{39,40}$ these were only adopted by several studies. ${ }^{16,17}$ Most included studies graded MR severity according to local institutional criteria, using a wide range of cutoffs for jet regurgitant area, vena contracta diameter, and/or proximal isovelocity surface area. This likely contributed to the considerable heterogeneity present in several of our analyses. To standardize comparative data across institutions, it is imperative that future studies use well-defined echocardiographic grading criteria according to established guidelines.

Results of the present meta-analysis were limited by several restraints, and should be interpreted with caution. First, there were only 4 RCTs, and observational studies 


\begin{tabular}{|c|c|c|c|c|c|c|c|c|}
\hline \multirow{2}{*}{$\begin{array}{l}\text { Study or Subgroup } \\
1.4 .1 \text { RCTs }\end{array}$} & \multicolumn{2}{|c|}{ CABG + MV Surgery } & \multicolumn{2}{|c|}{ Isolated CABG } & Weight & $\begin{array}{c}\text { Risk Ratio } \\
\mathrm{M}-\mathrm{H}, \text { Random, } 95 \% \mathrm{Cl}\end{array}$ & \multicolumn{2}{|c|}{$\begin{array}{c}\text { Risk Ratio } \\
\mathrm{M}-\mathrm{H}, \text { Random, } 95 \% \mathrm{Cl}\end{array}$} \\
\hline & & & & & & & & \\
\hline Fattouch 2009 & 0 & 45 & 29 & 48 & $4.5 \%$ & $0.02[0.00,0.29]$ & & \\
\hline Chan 2012 & 1 & 27 & 16 & 32 & $7.9 \%$ & $0.07[0.01,0.52]$ & & \\
\hline Smith 2014 & 16 & 140 & 43 & 140 & $25.4 \%$ & $0.37[0.22,0.63]$ & $\varpi$ & \\
\hline $\begin{array}{l}\text { Bouchard } 2014 \\
\text { Subtotal }(95 \% \mathrm{CI})\end{array}$ & 1 & $\begin{array}{r}13 \\
225\end{array}$ & 2 & $\begin{array}{r}14 \\
234\end{array}$ & $\begin{array}{r}6.2 \% \\
44.0 \%\end{array}$ & $\begin{array}{l}0.54[0.06,5.26] \\
0.16[0.04,0.75]\end{array}$ & & \\
\hline $\begin{array}{l}\text { Total events } \\
\text { Heterogeneity: Tau }{ }^{2} \\
\text { Test for overall effec }\end{array}$ & $\begin{array}{r}18 \\
1.54 ; \mathrm{Chi}^{2}= \\
Z=2.33(\mathrm{P}\end{array}$ & $\mathrm{df}=$ & $\begin{array}{r}90 \\
3(P=0 .\end{array}$ & $; I^{2}=6$ & $58 \%$ & & & \\
\hline
\end{tabular}

A

\begin{tabular}{|c|c|c|c|c|c|}
\hline \multicolumn{6}{|c|}{ 1.4.2 Observational Studies } \\
\hline Silberman 2011 & 2 & 51 & 32 & 68 & $12.6 \%$ \\
\hline Jeong 2012 & 4 & 59 & 11 & 45 & $16.3 \%$ \\
\hline Harris 2002 & 2 & 12 & 24 & 45 & $13.5 \%$ \\
\hline $\begin{array}{l}\text { Goland } 2009 \\
\text { Subtotal }(95 \% \mathrm{Cl})\end{array}$ & 2 & $\begin{array}{r}9 \\
131\end{array}$ & 11 & $\begin{array}{r}23 \\
181\end{array}$ & $\begin{array}{l}13.5 \% \\
56.0 \%\end{array}$ \\
\hline \multicolumn{6}{|c|}{$\begin{array}{l}\text { Heterogeneity: } \mathrm{Tau}^{2}=0.11 ; \mathrm{Chi}^{2}=3.79, \mathrm{df}=3(\mathrm{P}=0.29) ; \mathrm{I}^{2}=21 \% \\
\text { Test for overall effect: } Z=3.87(\mathrm{P}=0.0001)\end{array}$} \\
\hline Total $(95 \% \mathrm{Cl})$ & & 356 & & 415 & $100.0 \%$ \\
\hline $\begin{array}{l}\text { Total events } \\
\text { Heterogeneity: Tau } \\
\text { Test for overall effe } \\
\text { Test for subgroup }\end{array}$ & $\begin{array}{l}28 \\
\mathrm{hi}^{2}= \\
9(\mathrm{P} \\
\mathrm{Chi}^{2}\end{array}$ & $\begin{array}{l}8, \mathrm{df}= \\
0001) \\
25, \mathrm{df}\end{array}$ & $\begin{array}{l}168 \\
P=0\end{array}$ & $; 1^{2}=$ & $46 \%$ \\
\hline
\end{tabular}

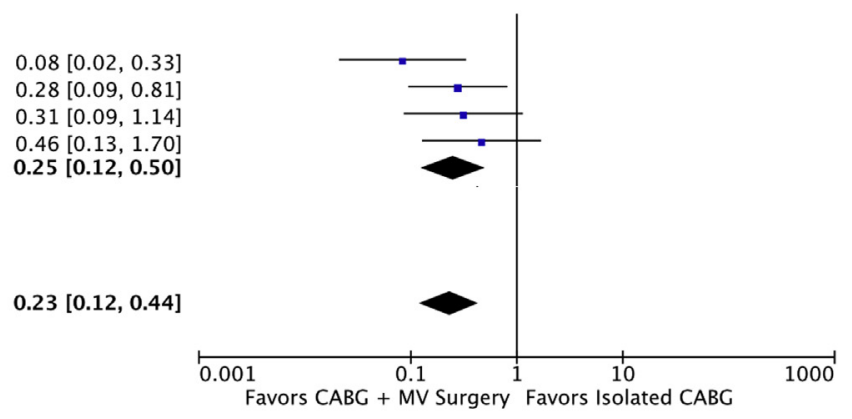

B

FIGURE 5. Forest plot displaying relative risk (RR) for recurrence of moderate-to-severe mitral regurgitation following isolated coronary artery bypass graft $(C A B G)$ surgery versus CABG and mitral valve $(M V)$ surgery in randomized controlled trials $(5 \mathrm{~A})$ and observational studies $(5 \mathrm{~B})$. The RR of individual studies corresponds to the middle of the squares, the horizontal lines show the $95 \%$ confidence intervals (CIs), and the pooled RR is represented by the middle of the solid diamond. A test of heterogeneity between studies is given below the summary statistics. RCT, Randomized controlled trial.

provided a significant proportion of clinical data. As observational studies did not use propensity-score matching or report adjusted estimates, it was not possible to account for potential baseline differences between the 2 surgical arms. In recognition of this limitation, we performed subgroup analyses by study design. Second, due to the lack of reporting, it was not possible to assess quality-oflife scores or measures of resource utilization and cost-effectiveness, such as length of intensive care unit stay, despite these being increasingly key considerations in clinical practice. Third, follow-up periods and endpoint definitions differed between institutions, and some studies may have underestimated adverse events due to incomplete follow-up. Although meta-regression analyses did not demonstrate significant interaction between follow-up duration and survival or residual MR, there was not enough data to perform meta-regression for other outcomes such as NYHA functional class. Last, due to the lack of available data, residual MR was assessed using a binary approach rather than through a longitudinal, competing risks method.

In conclusion, available data in the current literature demonstrated that MV surgery in addition to CABG was not associated with increased perioperative mortality in patients with moderate-to-severe IMR. Compared with isolated $\mathrm{CABG}$, concomitant MV surgery was associated with reduced recurrence of moderate-to-severe $\mathrm{MR}$ at follow-up. However, there was no significant difference between the 2 treatment approaches with regard to late mortality or NYHA functional status. Larger trials with longer follow-up duration are required to further assess overall survival and freedom from reintervention, as well as the impact of both surgical approaches on quality of life and health care costs.

\section{Conflict of Interest Statement}

Authors have nothing to disclose with regard to commercial support.

\section{References}

1. Bursi F, Enriquez-Sarano M, Jacobsen SJ, Roger VL. Mitral regurgitation after myocardial infarction: a review. Am J Med. 2006;119:103-12.

2. Aronson D, Goldsher N, Zukermann R, Kapeliovich M, Lessick J, Mutlak D, et al. Ischemic mitral regurgitation and risk of heart failure after myocardial infarction. Arch Intern Med. 2006;166:2362-8.

3. Grigioni F, Enriquez-Sarano M, Zehr KJ, Bailey KR, Tajik AJ. Ischemic mitral regurgitation long-term outcome and prognostic implications with quantitative Doppler assessment. Circulation. 2001;103:1759-64.

4. Kron IL, Acker MA, Adams DH, Ailawadi G, Bolling SF, Hung JW, et al. 2015 American Association for Thoracic Surgery (AATS) Consensus Guidelines: Ischemic mitral valve regurgitation. J Thorac Cardiovasc Surg. 2016;151: 940-56.

5. Nishimura RA, Otto CM, Bonow RO, Carabello BA, Erwin JP, Guyton RA, et al. American College of Cardiology/ American Heart Association Task Force on Practice Guidelines. 2014 AHA/ ACC guideline for the management of patients with valvular heart disease: a report of the American College of Cardiology/ American Heart Association Task Force on Practice Guidelines. J Thorac Cardiovasc Surg. 2014;148:e1-132.

6. Penicka M, Linkova H, Lang O, Fojt R, Kocka V, Vanderheyden M, et al. Predictors of improvement of unrepaired moderate ischemic mitral regurgitation in patients undergoing elective isolated coronary artery bypass graft surgery. Circulation. 2009;120:1474-81. 
7. Roshanali F, Mandegar MH, Yousefnia MA, Alaeddini F, Wann S. Low-dose dobutamine stress echocardiography to predict reversibility of mitral regurgitation with CABG. Echocardiography. 2006;23:31-7.

8. Aklog L, Filsoufi F, Flores KQ, Chen RH, Cohn LH, Nathan NS, et al. Does cor onary artery bypass grafting alone correct moderate ischemic mitral regurgitation? Circulation. 2001;104:I68-75.

9. Parmar MKB, Torri V, Stewart L. Extracting summary statistics to perform metaanalyses of the published literature for survival endpoints. Stat Med. 1998;17: 2815-34.

10. Tierney JF, Stewart LA, Ghersi D, Burdett S, Sydes MR. Practical methods for incorporating summary time-to-event data into meta-analysis. Trials. 2007;8:16.

11. Wan X, Wang W, Liu J, Tong T. Estimating the sample mean and standard deviation from the sample size, median, range and/or interquartile range. BMC Med Res Methodol. 2014;14:135

12. Begg CB, Mazumdar M. Operating characteristics of a rank correlation test for publication bias. Biometrics. 1994;50:1088-101.

13. Egger M, Smith GD, Schneider M, Minder C. Bias in meta-analysis detected by a simple, graphical test. BMJ. 1997;315:629-34.

14. Duval S, Tweedie R. Trim and fill: a simple funnel-plot-based method of testing and adjusting for publication bias in meta-analysis. Biometrics. 2000;56:455-63.

15. Bouchard D, Jensen H, Carrier M, Dermers P, Pellerin M, Perrault LP, et al. Effect of systematic downsizing rigid ring annuloplasty in patients with moderate ischemic mitral regurgitation. J Thorac Cardiovasc Surg. 2014;147:1471-7.

16. Smith PK, Puskas JD, Ascheim DD, Voisine P, Gelijns AC, Moskowitz AJ, et al. Surgical treatment of moderate ischemic mitral regurgitation. $N$ Engl J Med. 2014:371:2178-88

17. Chan KMJ, Punjabi PP, Flather M, Wage R, Symmonds K, Roussin I, et al. Coronary artery bypass surgery with or without mitral valve annuloplasty in moderate functional ischemic mitral regurgitation final results of the Randomized Ischemic Mitral Evaluation (RIME) trial. Circulation. 2012;126:2502-10.

18. Fattouch K, Guccione F, Sampognaro R, Panzarella G, Corrado E, Navarra E et al. POINT: efficacy of adding mitral valve restrictive annuloplasty to coronary artery bypass grafting in patients with moderate ischemic mitral valve regurgitation: a randomized trial. J Thorac Cardiovasc Surg. 2009;138:278-85.

19. Castleberry AW, Williams JB, Daneshmand MA, Honeycutt E, Shaw LK, Samad Z, et al. Surgical revascularization is associated with maximal survival in patients with ischemic mitral regurgitation: a 20-year experience. Circulation. 2014; $129: 2547-56$

20. Sá MP, Soares EF, Santos CA, Figueiredo OJ, Lima RO, Escobar RR, et al. Mitral valve replacement combined with coronary artery bypass graft surgery in patients with moderate-to-severe ischemic mitral regurgitation. Rev Port Cardiol. 2013; 32:131-7.

21. Deja MA, Grayburn PA, Sun B, Rao V, She L, Kreica M, et al. Influence of mitral regurgitation repair on survival in the surgical treatment for ischemic heart failure trial. Circulation. 2012;125:2639-48.

22. Jeong DS, Lee HY, Kim WS, Sung K, Park PW, Lee YT. Off pump coronary artery bypass versus mitral annuloplasty in moderate ischemic mitral regurgitation. Ann Thorac Cardiovasc Surg. 2012;18:322-30.

23. Silberman S, Eldar O, Oren A, Tauber R, Fink D, Klutstein MW, et al. Surgery for ischemic mitral regurgitation: should the valve be repaired. $J$ Heart Valve Dis. 2011;20:129-35.

24. de Waroux JB, Pouleur A-C, Vancraeynest D, Pasquet A, Gerber BL, El Khoury G, et al. Early hazards of mitral ring annuloplasty in patients with moderate to severe ischemic mitral regurgitation undergoing coronary revascularization: the importance of preoperative myocardial viability. J Heart Valve Dis. 2009:18:35-43.

25. Goland S, Czer LSC, Siegel RJ, DeRobertis MA, Mirocha J, et al. Outcomes in patients with moderate ischemic mitral regurgitation. Tex Heart Inst J. 2009;36: 416-24.
26. Mihaljevic T, Lam B-K, Rajeswaran J, Takagaki M, Lauer MS, Gillinov AM, et al. Impact of mitral valve annuloplasty combined with revascularization in patients with functional ischemic mitral regurgitation. J Am Coll Cardiol. 2007;49: 2191-201.

27. Bonacchi M, Prifti E, Maiani M, Frati G, Nathan NS, Leacche M. Mitral valve surgery simultaneous to coronary revascularization in patients with end-stage ischemic cardiomyopathy. Heart Vessels. 2006;21:20-7.

28. Buja P, Tarantini G, Del Bianco F, Razzolini R, Bilato C, Ramondo A, et al. Moderate-to-severe ischemic mitral regurgitation and multivessel coronary artery disease: impact of different treatment on survival and rehospitalization. Int $J$ Cardiol. 2006;111:26-33.

29. Kang D-H, Kim M-J, Kang S-J, Song JM, Song H, Hong MK, et al. Mitral valve repair versus revascularization alone in the treatment of ischemic mitral regurgitation. Circulation. 2006;114:I499-503

30. Kim Y-H, Czer LSC, Soukiasian HJ, De Robertis M, Magliato KE, Blanche C, et al. Ischemic mitral regurgitation: revascularization alone versus revascularization and mitral valve repair. Ann Thorac Surg. 2005:79:1895-901.

31. Wong DR, Agnihotri AK, Hung JW, Vlahakes GJ, Akins CW, Hilgenberg AD et al. Long-term survival after surgical revascularization for moderate ischemic mitral regurgitation. Ann Thorac Surg. 2005;80:570-7.

32. Diodato MD, Moon MR, Pasque MK, Barner HB, Moazami N, Lawton JS, et al. Repair of ischemic mitral regurgitation does not increase mortality or improve long-term survival in patients undergoing coronary artery revascularization: a propensity analysis. Ann Thorac Surg. 2004;78:794-9.

33. Trichon BH, Glower DD, Shaw LK, Cabell CH, Anstrom KJ, Felker GM, et al. Survival after coronary revascularization, with and without mitral valve surgery, in patients with ischemic mitral regurgitation. Circulation. 2003;108:II103-10.

34. Harris KM, Sundt TM, Aeppli D, Sharma R, Barzilai B. Can late survival of patients with moderate ischemic mitral regurgitation be impacted by intervention on the valve? Ann Thorac Surg. 2002;74:1468-75.

35. Despotis GJ, Filos KS, Zoys TN, Hogue CW Jr, Spitznagel E, Lappas DG. Fac tors associated with excessive postoperative blood loss and hemostatic transfusion requirements: a multivariate analysis in cardiac surgical patients. Anesth Analg. 1996;82:13-21.

36. Sablotzki A, Welters I, Lehmann N, Menges T, Gorlach G, Dehne M, et al. Plasma levels of immunoinhibitory cytokines interleukin-10 and transforming growth factor-b in patients undergoing coronary artery bypass grafting. Eur $J$ Cardiothorac Surg. 1997;11:763-8.

37. Taylor KM. Brain damage during cardiopulmonary bypass. Ann Thorac Surg 1998;65:S20-6

38. Di Salvo TG, Acker MA, Dec GW, Byrne JG. Mitral valve surgery in advanced heart failure. J Am Coll Cardiol. 2010;55:271-82.

39. Bonow RO, Carabello BA, Chatterjee K, de Leon AC, Faxon DP, Freed MD, et al. 2008 focused update incorporated into the ACC/AHA 2006 guidelines for the management of patients with valvular heart disease: a report of the American College of Cardiology/American Heart Association Task Force on Practice Guidelines (Writing Committee to revise the 1998 guidelines for the management of patients with valvular heart disease) Endorsed by the Society of Cardiovascular Anesthesiologists, Society for Cardiovascular Angiography and Interventions, and Society of Thoracic Surgeons. J Am Coll Cardiol. 2008;52 e1-42.

40. Zoghbi WA, Enriquez-Sarano M, Foster E, Grayburn PA, Kraft CD, Levine RA et al. Recommendations for evaluation of the severity of native valvular regurgitation with two-dimensional and Doppler echocardiography. J Am Soc Echocar diogr. 2003; 16:777-802

Key Words: ischemic mitral regurgitation, mitral valve repair, $\mathrm{CABG}$, meta-analysis 